\title{
Inside Dynamics of Delayed Traveling Waves
}

\author{
O. Bonnefon ${ }^{1}$, J. Garnier ${ }^{1,2}$, F. Hamel ${ }^{2,3}$, L. Roques ${ }^{1} *$ \\ ${ }^{1}$ UR 546 Biostatistique et Processus Spatiaux, INRA, 84000 Avignon, France \\ 2 Aix Marseille Université, CNRS, Centrale Marseille, LATP, UMR 7353, 13453 Marseille, France \\ ${ }^{3}$ Institut Universitaire de France
}

\begin{abstract}
The notion of inside dynamics of traveling waves has been introduced in the recent paper [14]. Assuming that a traveling wave $u(t, x)=U(x-c t)$ is made of several components $v^{i} \geq 0(i \in I \subset \mathbb{N})$, the inside dynamics of the wave is then given by the spatio-temporal evolution of the densities of the components $v^{i}$. For reaction-diffusion equations of the form $\partial_{t} u(t, x)=\partial_{x x} u(t, x)+f(u(t, x))$, where $f$ is of monostable or bistable type, the results in [14] show that traveling waves can be classified into two main classes: pulled waves and pushed waves. Using the same framework, we study the pulled/pushed nature of the traveling wave solutions of delay equations

$$
\partial_{t} u(t, x)=\partial_{x x} u(t, x)+F(u(t-\tau, x), u(t, x)) .
$$

We begin with a review of the latest results on the existence of traveling wave solutions of such equations, for several classical reaction terms. Then, we give analytical and numerical results which describe the inside dynamics of these waves. From a point of view of population ecology, our study shows that the existence of a non-reproductive and motionless juvenile stage can slightly enhance the genetic diversity of a species colonizing an empty environment.
\end{abstract}

Keywords and phrases: traveling waves, delayed equation, pulled and pushed solutions, Kobayashi's equation, Hutchinson's equation

Mathematics Subject Classification: 35C07, 35K57, 35Q92

\section{Introduction and main results}

This papers deals with the traveling wave solutions of delayed nonlinear parabolic equations of the form:

$$
\partial_{t} u(t, x)=\partial_{x x} u(t, x)+F(u(t-\tau, x), u(t, x)), \quad t>0, x \in \mathbb{R},
$$

where $\tau$ is a positive constant. These equations are generalizations of the classical reaction-diffusion equations:

$$
\partial_{t} u(t, x)=\partial_{x x} u(t, x)+f(u(t, x)), \quad t>0, x \in \mathbb{R} .
$$

The equation (1.2) arises in various scientific domains, from population dynamics $[9,17,32,35,39]$ to chemistry $[7,12]$. Throughout the paper, we assume that $f(0)=f(1)=0$. In the framework of population

${ }^{*}$ Corresponding author. E-mail: lionel.roques@avignon.inra.fr 
dynamics, for instance, the quantity $u$ stands for the population density and the nonlinear term $f(u(t, x))$ in (1.2) corresponds to the growth term.

In the delayed equation (1.1), the growth term $F(u(t-\tau, x), u(t, x))$ also depends on the quantity $u(t-\tau, x)$ at time $t-\tau$. This type of growth term has been introduced in ecological models of ordinary differential equations in [22] and can arise in many situations. A typical example is the existence of a non-reproductive and motionless juvenile stage. During the life cycle of many insects, for instance, the juvenile - or immature - stage consists of the egg, larva and pupa which cannot reproduce and do not move or move slowly compared to adults. In such cases, if the duration of the juvenile stage is $\tau$, the value of the growth term at time $t$ and position $x$ depends on the number of eggs which were laid at time $t-\tau$ at the position $x$. This number is a function of the density of adult individuals $u(t-\tau, x)$. The growth term can also depend on the current density of adult individuals, $u(t, x)$, because of crowding effects.

\subsection{The inside structure of the traveling waves for problem (1.2)}

Under some suitable assumptions on the function $f$, the problem (1.2) admits traveling wave solutions of the form $u(t, x)=U_{c}(x-c t)$, where $c>0$ is the speed of the wave and $U_{c}$ is a positive function which describes the profile of the wave. It satisfies the equation:

$$
\left\{\begin{array}{l}
U_{c}^{\prime \prime}(y)+c U_{c}^{\prime}(y)+f\left(U_{c}(y)\right)=0, \quad y \in \mathbb{R}, \\
U_{c}(-\infty)=1, \quad U_{c}(+\infty)=0 \text { and } 0<U_{c}<1 \text { on } \mathbb{R} .
\end{array}\right.
$$

The existence of such solutions, describing the invasion of the stationary state 0 by the state 1 , with a constant speed $c$ and a constant profile $U_{c}$, was one of the main reasons for the success of the reactiondiffusion theory in applied sciences. There is a huge literature on the properties of these traveling waves. In particular, existence results have been derived in $[3,4,12,23,25]$, uniqueness results (up to shift) are detailed in $[4,13]$, and the stability properties of the waves have been extensively analyzed in e.g. $[8,10,13,25,27,36,37,43]$. Some particular existence results will be recalled and used later for some specific nonlinearities $f$.

More recently, the notion of inside dynamics of traveling waves has been introduced in [14]. Assume that a traveling wave solution $u(t, x)=U_{c}(x-c t)$ of (1.2) is made of several neutral components $v^{i} \geq 0$ $(i \in I \subset \mathbb{N})$ which satisfy the equation

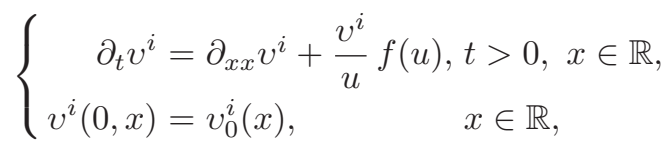

where the components $v^{i}$ are such that

$$
u(0, x)=\sum_{i \in I} v_{0}^{i}(x), \quad x \in \mathbb{R} .
$$

The inside dynamics of the wave

$$
u(t, x)=U_{c}(x-c t)=\sum_{i \in I} v^{i}(t, x)
$$

is then given by the spatio-temporal evolution of the densities of the components $v^{i}$. Notice that the equality $U_{c}(x-c t)=\sum_{i \in I} v^{i}(t, x)$ is a consequence of the fact that $u$ satisfies the same equation

$$
\partial_{t} u=\partial_{x x} u+\frac{u}{u} f(u)
$$

where $v^{i}$ has been replaced by $u$ in (1.3), as do all the components $v^{i}$. In this model, derived from [18,44], all components $v^{i}$ are neutral in the sense that they share the same characteristics as the front $u$ : they all 
have the same diffusion coefficient and the same per capita growth rate. The motivation of this model will be detailed later. Based on the formulation (1.3), it was shown in [14] that for the standard nonlinearities $f$ (monostable and bistable nonlinearities), the traveling wave solutions of (1.2) can then be classified into the following two types:

Definition 1.1 (Pulled wave). A traveling wave $u(t, x)=U_{c}(x-c t)$ is said to be a pulled wave if, for any component $v^{i}$ satisfying $(1.3), 0 \leq v_{0}^{i} \leq U_{c}$ and $v_{0}^{i}(x)=0$ for large $x$, there holds

$$
v^{i}(t, x+c t) \rightarrow 0 \text { as } t \rightarrow+\infty, \text { uniformly on compact sets. }
$$

Assume that $U_{c}$ is a pulled wave and consider the (right) component $v^{r}$, which satisfies at $t=0$, $v_{0}^{r}=U_{c} \cdot \mathbb{1}_{[\alpha,+\infty)}$, where $\mathbb{1}_{[\alpha,+\infty)}$ denotes the indicator function of the interval $[\alpha,+\infty)$, for some $\alpha \in \mathbb{R}$. The component $v^{l}$ corresponding to the remaining part of the wave satisfies $v_{0}^{l}=U_{c} \cdot \mathbb{1}_{(-\infty, \alpha)}$ and therefore converges to 0 in the moving frame with speed $c$. Since

$$
u(t, x+c t)=U_{c}(x)=v^{l}(t, x+c t)+v^{r}(t, x+c t),
$$

it follows that $v^{r}(t, x+c t)$ converges to $U_{c}(x)$ as $t \rightarrow \infty$ in the moving frame. Thus, the wave $U_{c}$ tends to be made of the rightmost component only: in other words, it is pulled by the component at the leading edge of the front.

Definition 1.2 (Pushed wave). A traveling wave $u(t, x)=U_{c}(x-c t)$ is said to be a pushed wave if, for any component $v^{i}$ satisfying (1.3), $0 \leq v_{0}^{i} \leq U_{c}$ and $v_{0}^{i} \not \equiv 0$, there exists $M>0$ such that

$$
\limsup _{t \rightarrow+\infty} \sup _{x \in[-M, M]} v^{i}(t, x+c t)>0 .
$$

This definition means that any component of a pushed wave propagates with the same speed $c$ as the total wave $U_{c}(x-c t)$. In other words, the wave is pushed by all its components.

In the KPP (for Kolmogorov, Petrovski and Piskunov) case, that is $f$ is positive in $(0,1)$ and such that $f(s) \leq f^{\prime}(0) s$ in $(0,1)$, there exists a minimal speed $c^{*}=2 \sqrt{f^{\prime}(0)}>0$ such that (1.2) admits traveling wave solutions with speed $c$ if and only if $c \geq c^{*}[25]$. The results in [14] show that in this case all the traveling wave solutions are pulled. In the general monostable case, that is when $f>0$ in $(0,1)$, there still exists a positive minimal speed $c^{*} \geq 2 \sqrt{f^{\prime}(0)}$ such that (1.2) admits traveling wave solutions with speed $c$ if and only if $c \geq c^{*}$. Note that the KPP assumption may not be satisfied. The results in [14] show that the critical traveling wave with speed $c^{*}$ is pushed if $c^{*}>2 \sqrt{f^{\prime}(0)}$ and is pulled otherwise. Furthermore, the supercritical waves with speeds $c>c^{*}$ are always pulled. On the other hand, in the bistable case, that is $f^{\prime}(0)<0, f^{\prime}(1)<0, \int_{0}^{1} f(s) d s>0$ and there exists $\rho \in(0,1)$ such that $f<0$ in $(0, \rho)$ and $f>0$ in $(\rho, 1)$, the unique traveling wave (see $[3,4,13]$ for the existence and uniqueness of this wave) is pushed [14] (notice that the speed $c$ is positive since $\int_{0}^{1} f(s) d s>0$ ).

The above definitions of pulled and pushed waves agree with the pulled/pushed terminology introduced by Stokes [40] in the monostable case. The definition of [40] was indeed based on the comparison between $c^{*}$ and $2 \sqrt{f^{\prime}(0)}$. If $c^{*}=2 \sqrt{f^{\prime}(0)}$, the minimal speed only depends on the linearization of the equation around the limiting state 0 and the critical wave with speed $c^{*}=2 \sqrt{f^{\prime}(0)}$ is then pulled by the leading edge of its tail. Otherwise, the critical wave with speed $c^{*}>2 \sqrt{f^{\prime}(0)}$ is pushed by its nonlinear part. The reason why the supercritical waves with speeds $c>c^{*}$ are pulled is not so obvious, apart from the known fact that they all have fat exponential tails as they converge to the unstable state 0 [4].

\subsection{Motivation and main assumptions for problem (1.1)}

For the equation (1.2) without delay, the new formulation of pushed and pulled solutions given in Definitions 1.1 and 1.2 had the advantage of being intuitive and to work in both the monostable and the bistable cases. Furthermore, it can easily be extended to more general models, as shown here for equation 
(1.1) with delay. In our setting, the pulled/pushed nature of the waves also gives a precise understanding of their inside dynamics. The comprehension of the dependence of the inside structure of the waves on the nonlinearity $f$ has profound implications in population genetics [34]. From this perspective, $u$ is interpreted as a density of genes, and the components $v^{i}$ correspond to neutral genetic fractions. Pulled waves indicate a strong erosion of the genetic diversity, while pushed waves indicate that the full genetic diversity of a population is conserved in the colonization front. As emphasized in [34], bistable nonlinearities correspond to a negative fertility at low densities, a phenomenon known as the Allee effect in ecological sciences [6]. The pushed nature of the wave in this case shows that the Allee effect has advantageous consequences on genetic diversity compared to the KPP case. This result is in contrast with previous studies on the Allee effect, which generally shows adverse consequences of the Allee effect. This perspective is inherited from demographic studies, which demonstrate that the Allee effect can lead to the failure of biological invasions $[15,28,29]$.

In the ecological literature, the existence of a juvenile stage has been suspected to lead to a better conservation of the genetic diversity [5]. The existence of a delay in (1.1) is also well known to reduce the minimal speed of traveling waves for KPP-type nonlinearities [38]. Thus, as for the Allee effect, we can expect that the introduction of a delay term in (1.1) will modify the inside structure of the waves. The main goal of this paper is to analyze the evolution of the inside structure of the solutions of the delayed equation (1.1). We focus on two main types of functions $F$ which have been considered in the literature: the Kobayashi and Hutchinson's types.

Kobayashi's equation. In this case, we assume that the function $F$ is of class $C^{1}([0,1] \times[0,1])$ and that it satisfies

$$
F(r, s)=f_{1}(r) f_{2}(s) \text {, for all } 0 \leq r, s \leq 1 .
$$

The function $f_{1}$ corresponds to the intrinsic growth rate of the population, i.e., the growth rate in the absence of saturation effects. The function $f_{2}$ describes the saturation of the environment. The term $F(u(t-\tau, x), u(t, x))$ in (1.1) therefore reflects the birth of new individuals from individuals which were present at time $t-\tau$, compensated by saturation effects depending on the present population density $u(t, x)$. More precisely, the functions $f_{1}$ and $f_{2}$ are assumed to satisfy

$$
\left\{\begin{array}{l}
\text { (i) } f_{1}, f_{2} \in C^{1}([0,1]) \\
\text { (ii) } f_{1}(0)=f_{2}(1)=0, f_{2}(0)=1 \\
\text { (iii) } f_{1}, f_{2}>0 \text { in }(0,1) \\
\text { (iv) } \beta:=f_{1}^{\prime}(0)>0 \text { and } 0 \leq f_{1}^{\prime}(r) \leq f_{1}^{\prime}(0) \text { for } r \in[0,1] \\
\text { (v) } f_{2}^{\prime}(s) \leq 0 \text { for } s \in[0,1] .
\end{array}\right.
$$

Under these assumptions, the function $f(r)=F(r, r)$ is of the KPP type: $f$ is positive in $(0,1)$ and satisfies $f(0)=f(1)=0$ and $0<f(r) \leq f^{\prime}(0) r$ for all $r \in(0,1)$. Thus, in the absence of delay $(\tau=0)$, the equation (1.1) is equivalent to the equation (1.2) with a KPP nonlinearity and traveling wave solutions $u(t, x)=U_{c}(x-c t)$ with $U_{c}(+\infty)=0<U_{c}<U_{c}(-\infty)=1$ exist if and only if $c \geq c^{*}(0):=2 \sqrt{\beta}$. A typical example of a function $F$ satisfying the assumptions (1.4) and (1.5) is $F(r, s)=r(1-s)$. The equation (1.1) with the nonlinearity $F(u(t-\tau, x), u(t, x))=u(t-\tau, x)(1-u(t, x))$ is known as Kobayashi's equation, and was derived in [24] from a branching process. Since then, several papers have been devoted to the analysis of the mathematical properties of this equation, with a special focus on its traveling wave solutions $[33,38]$.

Hutchinson's equation. Another typical example of function $F$ in (1.1) is:

$$
F(r, s)=s(1-r) .
$$

This nonlinear term was first considered in the work of Hutchinson [22], and the equation (1.1) is thereby called the Hutchinson's equation. This is the most classical example of reaction-diffusion equation with 
temporal delay. Here, the assumption (1.5) is not satisfied. Moreover, since the nonlinear term $u(t, x)(1-$ $u(t-\tau, x))$ is nonmonotone with respect to the delay argument, the proof of the existence of traveling waves is more involved in this case than for Kobayashi's equation.

From an ecological viewpoint, the equation (1.1) with the nonlinear term (1.6) means that the intrinsic growth rate depends on the present population density $u(t, \cdot)$, whereas the saturations effects depend on the past population density $u(t-\tau, \cdot)$.

\subsection{Traveling waves for the delayed equation (1.1)}

As in the no-delay case, a traveling wave solution of (1.1) propagating with the speed $c>0$ can be defined as an entire solution of (1.1) of the form $u(t, x)=U_{c}(x-c t)$, where $U_{c}>0$ satisfies the delayed ordinary differential equation:

$$
\left\{\begin{array}{l}
U_{c}^{\prime \prime}(y)+c U_{c}^{\prime}(y)+F\left(U_{c}(y+c \tau), U_{c}(y)\right)=0, \quad y \in \mathbb{R} \\
U_{c}(-\infty)=1, \quad U_{c}(+\infty)=0
\end{array}\right.
$$

For Kobayashi's equation, that is when $F$ satisfies (1.4)-(1.5), Schaaf $[38]^{1}$ has proved the existence of a minimal speed $c^{*}(\tau)>0$, depending only on $\beta:=f_{1}^{\prime}(0)$ and $\tau$, such that the equation (1.1) has no traveling wave solution if $c<c^{*}(\tau)$, whereas it admits a unique (up to shifts in $y$ ) traveling wave solution with speed $c$ for any $c>c^{*}(\tau)$ (see also Theorem 5.1 of [30]). Besides, the traveling wave solutions are decreasing. The case $c=c^{*}(\tau)$ can be treated as in Theorem 4.6 of [33]: traveling wave solutions with the speed $c^{*}(\tau)$ exist, they are decreasing and they are unique up to shifts. Some explicit bounds on $c^{*}(\tau)$ with respect to the delay $\tau$ can be found in [49].

In the Hutchinson case, that is when $F$ satisfies (1.6), the equation (1.1) still admits traveling wave solutions when the delay $\tau$ is small and when $c$ is larger than the minimal speed $c_{H}^{*}=2$. More precisely, it is proved in [16] that monotone traveling waves $\left(c, U_{c}\right)$ satisfying $(1.7)$ exist if and only if

$$
\left\{\begin{array}{l}
0 \leq \tau \leq e^{-1} \simeq 0.37 \text { and } c \in[2, \infty), \\
\text { or } \\
e^{-1}<\tau \leq h_{1} \simeq 0.56 \text { and } c \in\left[2, c^{\sharp}(\tau)\right] .
\end{array}\right.
$$

The precise definitions of $c^{\sharp}(\tau)<\infty$ and $h_{1}$ are given in [16] (see also [26]). For any delay $\tau$ and any speed $c$, these monotone fronts are unique up to translation [20].

When the conditions (1.8) are not satisfied, the recent study [20] also proves the existence of nonmonotone waves satisfying the equation (1.7), for any $c \geq 2$, when $e^{-1}<\tau \leq 1$. For further existence and qualitative results for small $\tau$ or large speeds with other types of nonlinearities, including the case of spatially nonlocal delays, we refer to $[1,2,11,26,31,41,42,45,46,48]$.

\subsection{Decomposition of the delayed traveling waves into neutral components}

As explained in Section 1.1 in the no-delay case, in order to study the dynamics of the inside structure of the waves, we assume that $u$ is initially (i.e., here, for $t \in[-\tau, 0]$ ) composed of different groups $\left(v_{0}^{i}\right)_{i \in I}$ such that, for every $i \in I$,

$$
v_{0}^{i} \in L^{\infty}(\mathbb{R}) \backslash\{0\} \text { and } 0 \leq v_{0}^{i}(x) \leq U_{c}(x) \text { for all } x \in \mathbb{R},
$$

where $I$ is a subset of $\mathbb{N}$ and

$$
u(0, x)=U_{c}(x)=\sum_{i \in I} v_{0}^{i}(x) \text { for all } x \in \mathbb{R} .
$$

As in (1.3), the components $v^{i}$ are assumed to be neutral in the sense that they diffuse and grow with the same manner inside the front $u(t, x)$. In the no-delay case, following [18,44], the growth term in the

\footnotetext{
${ }^{1}$ In [38], the assumption $f_{1}^{\prime}(r) \leq f_{1}^{\prime}(0)$ was not explicitly written, although it is used in the proof.
} 
equation (1.3) satisfied by the components was given by the proportion $\left(v^{i} / u\right) f(u)$ of the total growth term $f(u)$.

Kobayashi case. Here, because of the delay $\tau$, and under our assumptions (1.4) and (1.5), each component grows proportionally to the delayed proportion $v^{i}(t-\tau, \cdot) / u(t-\tau, \cdot)$ of the global growth term $F(u(t-$ $\tau, x), u(t, x))$. The component densities therefore satisfy the equation

$$
\left\{\begin{aligned}
\partial_{t} v^{i}(t, x) & =\partial_{x x} v^{i}(t, x)+\frac{v^{i}(t-\tau, x)}{u(t-\tau, x)} F(u(t-\tau, x), u(t, x)), & t>0, x \in \mathbb{R}, \\
v^{i}(t, x) & =v_{0}^{i}(x-c t), & t \in[-\tau, 0], x \in \mathbb{R} .
\end{aligned}\right.
$$

In other words, the growth rate of each component is proportional to its density at time $t-\tau$ but the per capita growth term $F(u(t-\tau, x), u(t, x)) / u(t-\tau, x)$ is the same for all components. The initial condition means that the inside structure of the wave remains unchanged when $t \in[-\tau, 0]$.

Hutchinson case. In this case, as in the no-delay case, each component grows in a proportion $v^{i}(t, \cdot) / u(t, \cdot)$ of the global growth term:

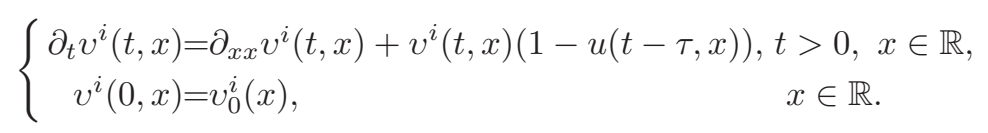

In both the Kobayashi and Hutchinson cases, it follows from the uniqueness of the solution that $u$ is the sum of all its components at positive times, that is

$$
u(t, x)=\sum_{i \in I} v^{i}(t, x) \text { for all } t>0 \text { and } x \in \mathbb{R} .
$$

The maximum principle also leads to the inequalities

$$
0<v^{i}(t, x) \leq u(t, x) \text { for all } t>0, x \in \mathbb{R} \text { and } i \in I .
$$

The first inequality $0<v^{i}(t, x)$ is a consequence of Proposition 2.2 in [38] in the Kobayashi case and of the classical strong parabolic maximum principle in Hutchinson case. The second inequality $v^{i}(t, x) \leq u(t, x)$ follows from the first one and (1.12).

\subsection{Main result: dynamics of the neutral components}

The initial decomposition (1.9) of the front into a sum of different labels is actually arbitrary and of course not unique. But what turns out to be uniquely determined by the front is the way these inside components behave at large time.

Theorem 1.3. 1 (Kobayashi case). Assume that $F$ satisfies (1.4)-(1.5). Let $\tau>0$ and let $\left(c, U_{c}\right)$ be a traveling wave solving (1.7), with $c>c^{*}(\tau)$. If the support of $v_{0}$ is in $(-\infty, M]$ for some $M \in \mathbb{R}$, then the solution $v$ of (1.10) satisfies:

$$
\max _{x \geq\left(c^{*}(\tau)+\delta\right) t} v(t, x) \rightarrow 0 \text { as } t \rightarrow+\infty \text { for all } \delta>0,
$$

and, in particular, $v(t, x+c t) \rightarrow 0$ as $t \rightarrow+\infty$ locally uniformly with respect to $x \in \mathbb{R}$.

2 (Hutchinson case). Assume that $F$ satisfies (1.6). Let $\tau>0$ and let $\left(c, U_{c}\right)$ be a traveling wave solving (1.7), with $c \geq 2$. If the support of $v_{0}$ is in $(-\infty, M]$ for some $M \in \mathbb{R}$, then the solution $v$ of (1.11) satisfies:

$$
\max _{x \geq A+2 t} v(t, x) \rightarrow 0 \text { as } t \rightarrow+\infty \text { for all } A \in \mathbb{R} .
$$

Theorem 1.3, which is proved in Section 3.1, means that the waves $\left(c, U_{c}\right)$, with $c>c^{*}(\tau)$ in the Kobayashi case and $c \geq 2$ in the Hutchinson case, are pulled by their rightmost components in the same sense as Definition 1.1. Note that, in the Kobayashi case, Theorem 1.3 does not show that the wave with minimal speed $c=c^{*}(\tau)$ is pulled. However, our numerical computations of Section 2 suggest that the wave $\left(c^{*}(\tau), U_{c^{*}(\tau)}\right)$ is also a pulled wave. 


\subsection{Transient transport phenomenon in the Kobayashi case}

Assume that $F$ satisfies (1.4)-(1.5). Let $\tau \geq 0$ and let $\left(c, U_{c}\right)$ be a traveling wave solving (1.7), with $c>c^{*}(\tau)$. In order to understand the dynamics of a component $v$ solving (1.10), inside the traveling wave solution, it is natural to make the following change of variables:

$$
\tilde{v}(t, x)=v(t, x+c t) \text { for all } t \geq 0 \text { and } x \in \mathbb{R} .
$$

The function $\tilde{v}$ corresponds to the solution $v$ in the moving frame at speed $c$. It obeys the following equation:

$$
\left\{\begin{aligned}
\partial_{t} \tilde{v}(t, x) & =\partial_{x}^{2} \tilde{v}(t, x)+c \partial_{x} \tilde{v}(t, x)+\frac{F\left(U_{c}(x+c \tau), U_{c}(x)\right)}{U_{c}(x+c \tau)} \tilde{v}(t-\tau, x+c \tau), t>0, x \in \mathbb{R}, \\
\tilde{v}(t, x) & =v_{0}(x), t \in[-\tau, 0], x \in \mathbb{R} .
\end{aligned}\right.
$$

Let us now look at the proportion $p$ of the total wave $U_{c}(x)$ corresponding to the component $\tilde{v}$. This quantity is defined by the ratio $p(t, x):=\tilde{v}(t, x) / U_{c}(x)$. It satisfies for all $t>0$ and $x \in \mathbb{R}$ :

$$
\partial_{t} p(t, x)=\partial_{x}^{2} p(t, x)+V(x) \partial_{x} p(t, x)+\frac{F\left(U_{c}(x+c \tau), U_{c}(x)\right)}{U_{c}(x)}(p(t-\tau, x+c \tau)-p(t, x)),
$$

with the (non-conservative) advection term:

$$
V(x):=c+2 \frac{U_{c}^{\prime}(x)}{U_{c}(x)} .
$$

When $\tau=0$, the reaction term in (1.13) vanishes. Moreover, under the KPP assumptions (1.4)-(1.5) on $F$, it is known [4] that $V(x)$ is positive for large $x\left(\right.$ since $\lim _{x \rightarrow+\infty} V(x)=\sqrt{c^{2}-4 \beta}>0$ when $\left.c>c^{*}(0)=2 \sqrt{\beta}\right)$. Therefore, the proportion $p$ is transported to the left in the moving frame. In this case, the wave $\left(c, U_{c}\right)$ is pulled in the sense of Definition 1.1, as shown in [14]. On the other hand, in absence of delay and if $f(r):=F(r, r)$ is of bistable type, then $V(x)$ is negative for large $x$ (since $\left.\lim _{x \rightarrow+\infty} V(x)=-\sqrt{c^{2}-4 f^{\prime}(0)}<0\right)$. The advection term pushes the proportion $p$ to the right and leads to a pushed wave, as shown in [14]. Thus, when $\tau=0$, the pulled/pushed nature of the waves is completely determined by the sign of $V(x)$ when $x \rightarrow+\infty$.

When $\tau>0$, there is a competition between the advection, diffusion and reaction terms in (1.13). This balance leads to pulled waves in all cases $c>c^{*}(\tau)$, as shown by part 1 of Theorem 1.3. However, when $c$ is below a certain threshold $\bar{c}(\tau)$, the advection term $V(x)$ is negative for large $x$ :

Proposition 1.4. Let $F$ satisfy (1.4)-(1.5) and let $\tau>0$. There exists $\bar{c}(\tau) \in\left(c^{*}(\tau),+\infty\right)$ such that, for any traveling wave $\left(c, U_{c}\right)$ solving $(1.7)$, there holds

$$
\left\{\begin{array}{l}
\lim _{x \rightarrow+\infty} V(x)<0 \text { if } c^{*}(\tau)<c<\bar{c}(\tau), \\
\lim _{x \rightarrow+\infty} V(x)=0 \text { if } c=\bar{c}(\tau), \\
\lim _{x \rightarrow+\infty} V(x)>0 \text { if } c>\bar{c}(\tau) .
\end{array}\right.
$$

When $c^{*}(\tau)<c<\bar{c}(\tau)$, the advection term $V(x)$ is oriented in the same direction as that of the wave for large $x$. This leads to a transient transport phenomenon to the positive $x$-direction in the moving frame, as shown in the numerical computations of Section 2. However, the effect of the advection term is dominated by that of the diffusion and reaction terms of (1.13) at large times in the moving frame, since $p(t, x)$ eventually converges to 0 as $t \rightarrow+\infty$ locally uniformly in $x \in \mathbb{R}$ by Theorem 1.3. Lastly, we conjecture that the inequality $\lim _{x \rightarrow+\infty} V(x)<0$ also holds true when $c=c^{*}(\tau)$ (see Lemma 3.1).

The next lemma shows that the length of the interval $\left(c^{*}(\tau), \bar{c}(\tau)\right)$ is a nonmonotone function of $\tau$.

Lemma 1.5. The length $\left|\bar{c}(\tau)-c^{*}(\tau)\right|$ of the interval $\left(c^{*}(\tau), \bar{c}(\tau)\right)$ converges to $\left|\bar{c}\left(0^{+}\right)-c^{*}\left(0^{+}\right)\right|=0$ as $\tau \rightarrow 0^{+}$, and to $\left|\bar{c}(+\infty)-c^{*}(+\infty)\right|=0$ as $\tau \rightarrow+\infty$.

The proofs of Proposition 1.4 and Lemma 1.5 are given in Section 3.2. 


\section{Numerical computations}

In order to study the inside dynamics of the traveling wave solutions of delayed equations of the form (1.1), we consider the case of a wave $\left(c, U_{c}\right)$ made of a finite number of components $v^{i}$ where $i=1, \ldots, N=7$. We assume that these components are defined at $t=0$ by $v_{0}^{1}=U_{c} \cdot \mathbb{1}_{\left(-\infty, x_{1}\right]}, v_{0}^{k}=U_{c} \cdot \mathbb{1}_{\left(x_{k-1}, x_{k}\right]}$ for $k=2, \ldots, N-1$ and $v_{0}^{N}=U_{c} \cdot \mathbb{1}_{\left(x_{N-1},+\infty\right)}$, and for the sequence $x_{1}<x_{2}<\ldots<x_{N-1}$ of equally spaced points (see Figs. 1-3). We numerically solved the equations satisfied by the components $v^{i}$ with four types of reaction terms. In all cases, the point $x_{N}$ which corresponds to the beginning of the rightmost component is such that $U_{c}\left(x_{N-1}\right)=0.1$.

\subsection{Reaction terms}

In order to investigate the effect of the delay $\tau$ on the inside dynamics of the waves, we begin with a classical KPP nonlinearity without delay:

$$
F_{1}(u(t-\tau, x), u(t, x))=u(t, x)(1-u(t, x)) .
$$

The second type of nonlinearity that we consider is of Kobayashi type:

$$
F_{2}(u(t-\tau, x), u(t, x))=u(t-\tau, x)(1-u(t, x)) .
$$

The third type of nonlinearity is of Hutchinson type:

$$
F_{3}(u(t-\tau, x), u(t, x))=u(t, x)(1-u(t-\tau, x)) .
$$

Lastly, we consider a Huxley [21] nonlinearity with delay (see [38, 47]):

$$
F_{4}(u(t-\tau, x), u(t, x))=u(t, x)(1-u(t, x))(u(t-\tau, x)-\rho),
$$

for some $\rho \in(0,1 / 2)$ (in our computations, we took $\rho=0.3)$. This last type of nonlinearity, which neither satisfies Kobayashi's assumptions (1.4)-(1.5) nor Hutchinson's assumption (1.6), was not investigated in the previous section of this manuscript. In the case $\tau=0$, it corresponds to a bistable nonlinearity and, as mentioned in Section 1.1, the corresponding wave is unique and is a pushed wave. Numerical computations have already been carried out in this case $\tau=0$ in [34]. In the case $\tau>0$, Theorem 3.13 in [38] implies that there exists a unique traveling wave $\left(c_{4}, U_{4}\right)$ solving $(1.7)$ in this case, and this wave is monotone. From the point of view of population ecology the reaction term $F_{4}$ corresponds to a sort of Allee effect: when $u \in(0,1)$, the growth term is positive if and only if the population density at time $t-\tau$ is larger than the threshold $\rho$. Although this assumption may appear unrealistic, our main goal here is to exhibit a numerical example of pushed wave in the context of delayed equations, and to compare it with the pulled waves corresponding to the reaction terms $F_{k}, k=1,2,3$. For this last type of reaction term $\left(F=F_{4}\right)$, the dynamics of the components $v^{i}$ are described by

$$
\left\{\begin{aligned}
\partial_{t} v^{i}(t, x) & =\partial_{x x} v^{i}(t, x)+v^{i}(t, x)(1-u(t, x))(u(t-\tau, x)-\rho), t>0, x \in \mathbb{R}, \\
v^{i}(0, x) & =v_{0}^{i}(x), \quad x \in \mathbb{R}
\end{aligned}\right.
$$

so that each component grows in a proportion $v^{i}(t, \cdot) / u(t, \cdot)$ of the global reaction term.

\subsection{Results}

The Figs. 1-3 show the evolution of the spatial structure of some traveling wave solutions of $æ(1.1)$ for the above-mentioned reaction terms. Each component is depicted with a different color and with a thickness which corresponds, at each position $x$, to the density $v^{i}$ of the component. In the numerical simulations, the speeds of the various traveling fronts are different. However, in order to be able to compare quantitatively the inside dynamics of the various fronts, the simulations are all stopped when the fronts reach approximately the same position (around $x=120$ ). 


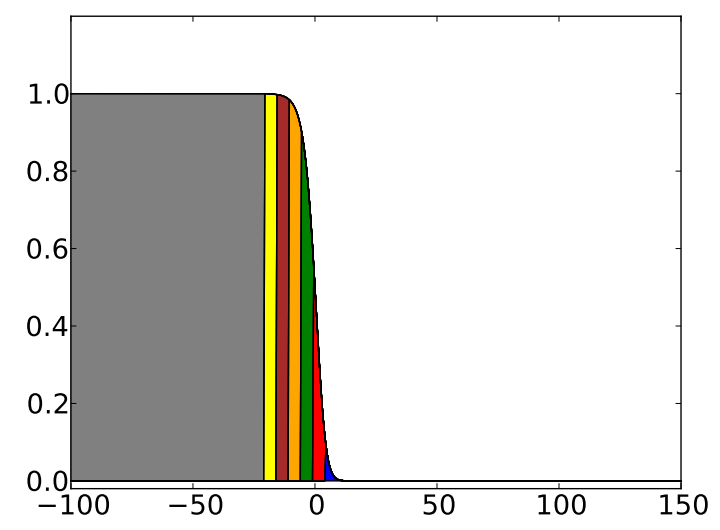

(a) KPP, $t=0$

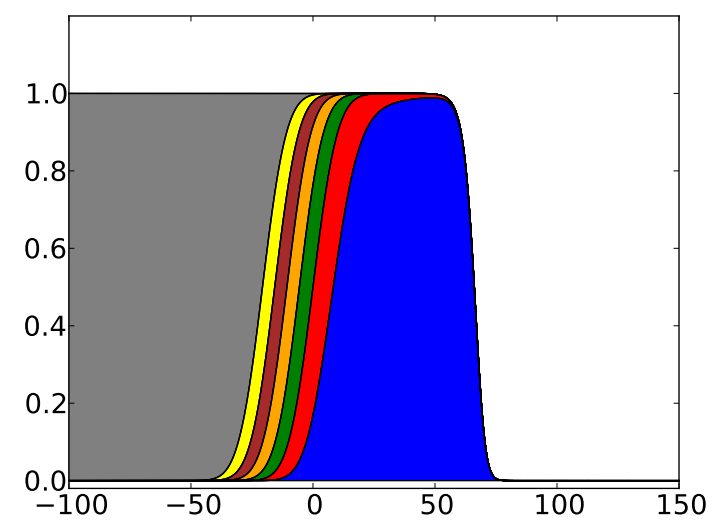

(c) $\mathrm{KPP}, t=30$

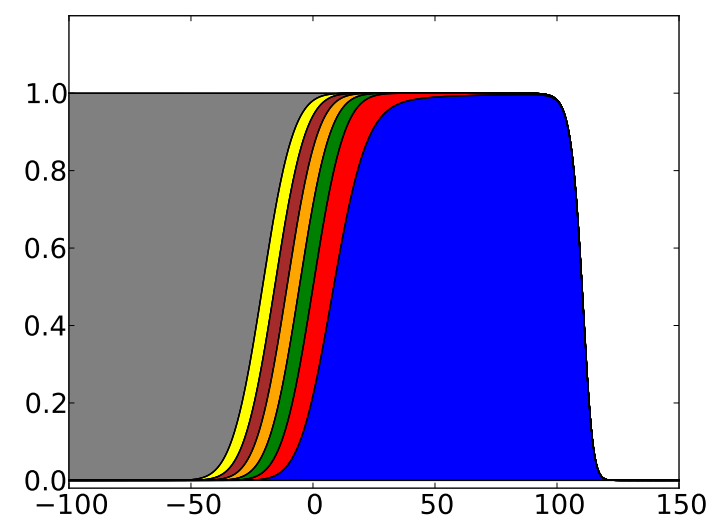

(e) $\mathrm{KPP}, t=50$

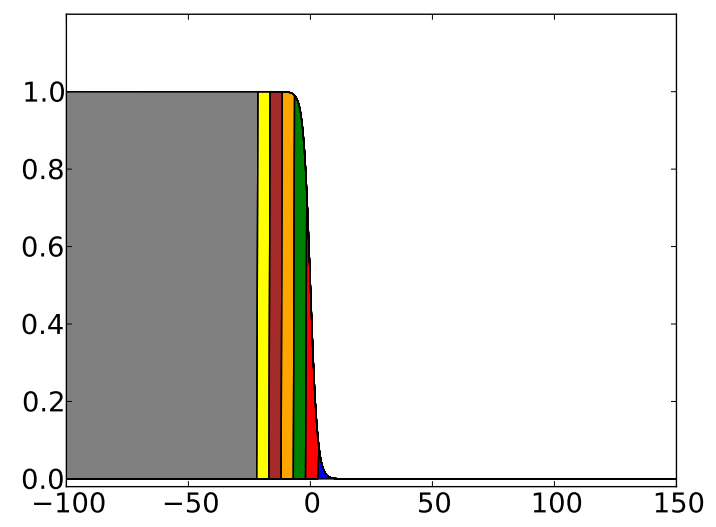

(b) Huxley, $t=0$

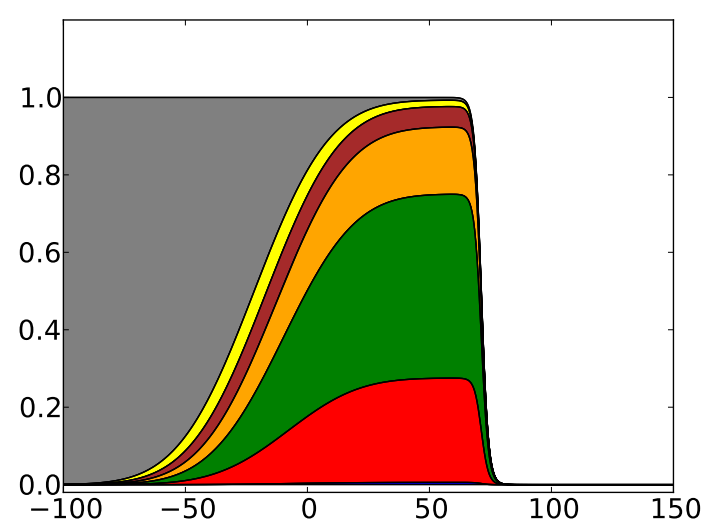

(d) Huxley, $t=300$

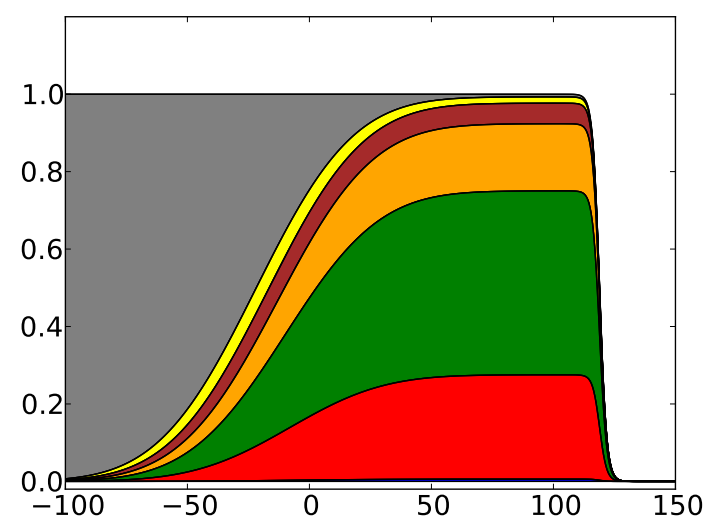

(f) Huxley, $t=500$

Figure 1: Inside dynamics of $\operatorname{KPP}\left(c=c^{*}(0)=2, \tau=0\right)$ and Huxley $\left(c=c_{4} \simeq 0.24, \tau=1\right)$ waves. Each component is depicted with a different color and with a thickness which corresponds, at each position $x$, to the density $v^{i}$ of the component. 


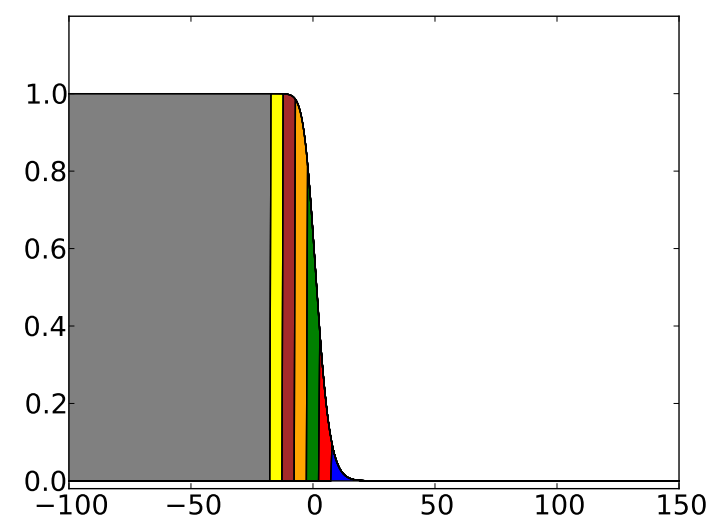

(a) Kobayashi, $c=c^{*}(10), t=0$

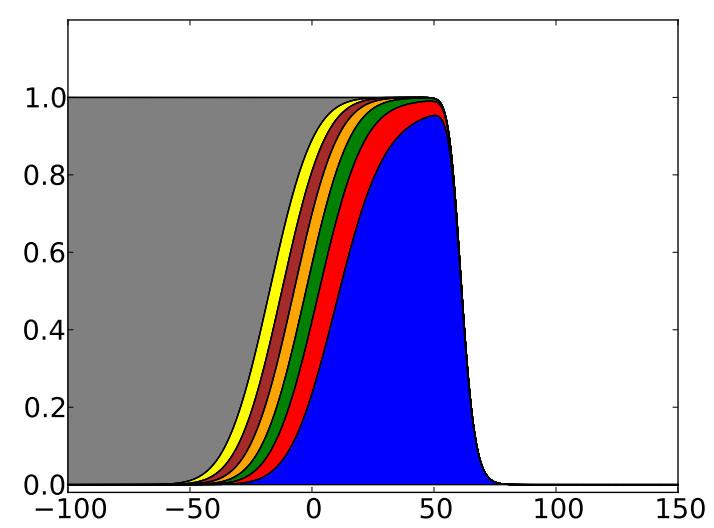

(c) Kobayashi, $c=c^{*}(10), t=100$

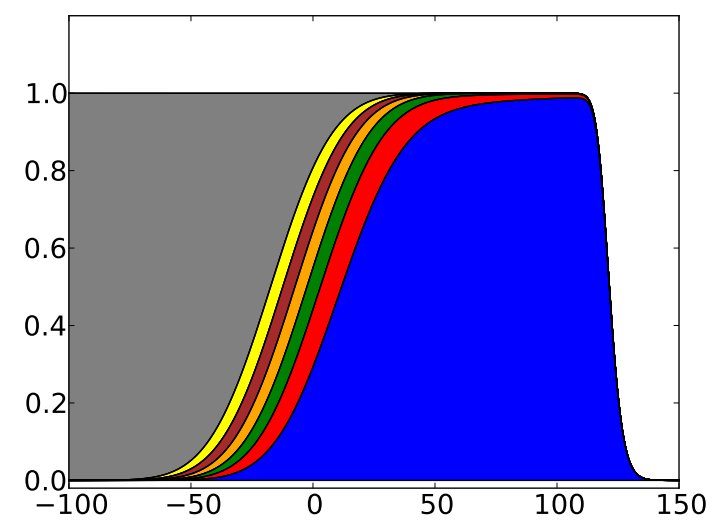

(e) Kobayashi, $c=c^{*}(10), t=200$

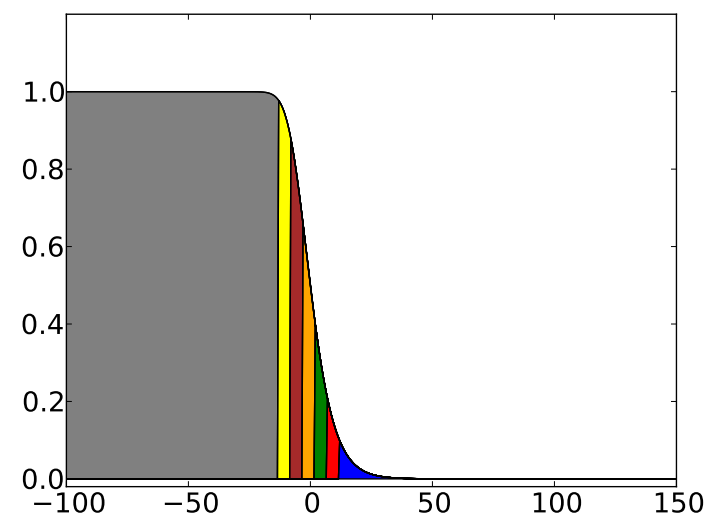

(b) Kobayashi, $c>\bar{c}(10), t=0$

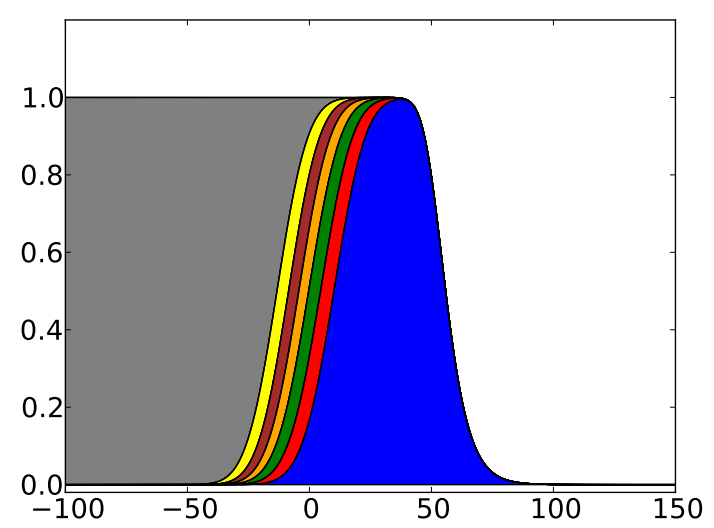

(d) Kobayashi, $c>\bar{c}(10), t=50$

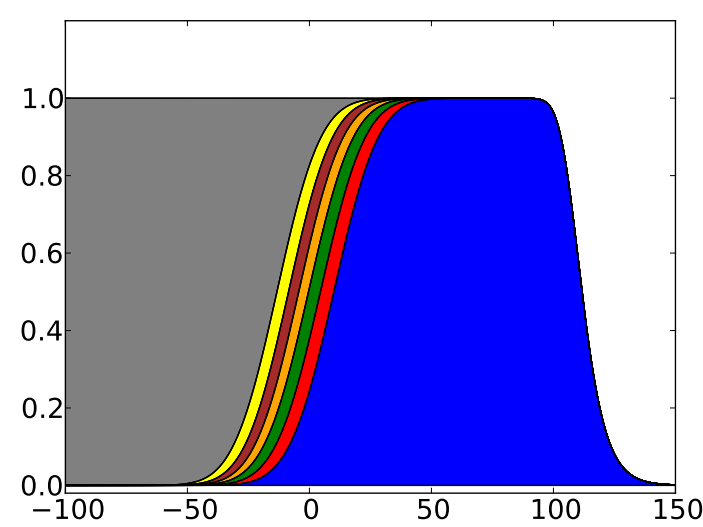

(f) Kobayashi, $c>\bar{c}(10), t=100$

Figure 2: Inside dynamics of Kobayashi waves. Left: $c=c^{*}(10) \simeq 0.60, \tau=10$; right: $c \simeq 1.11>\bar{c}(10) \simeq$ $0.66, \tau=10$. 


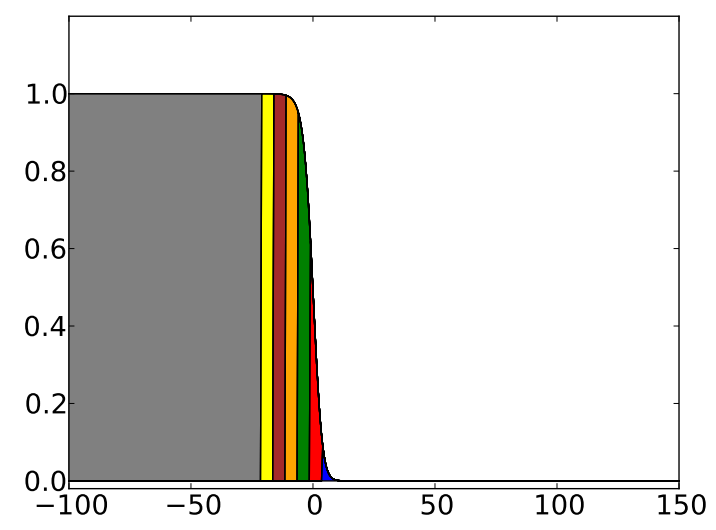

(a) Hutchinson $(\tau=0.3), t=0$

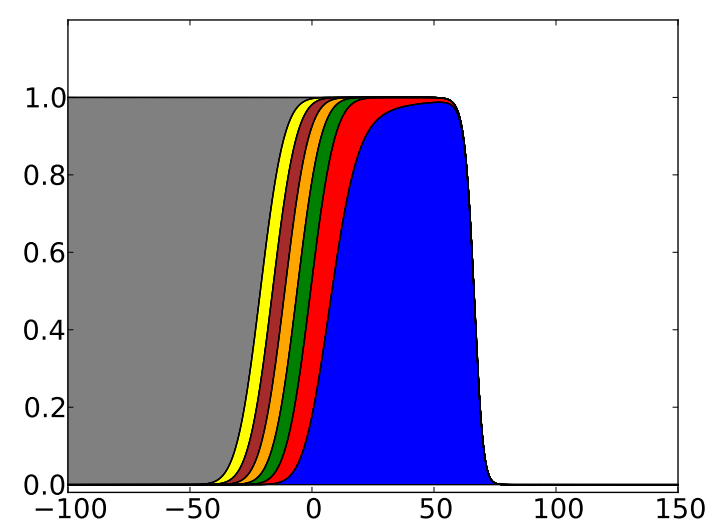

(c) Hutchinson $(\tau=0.3), t=30$

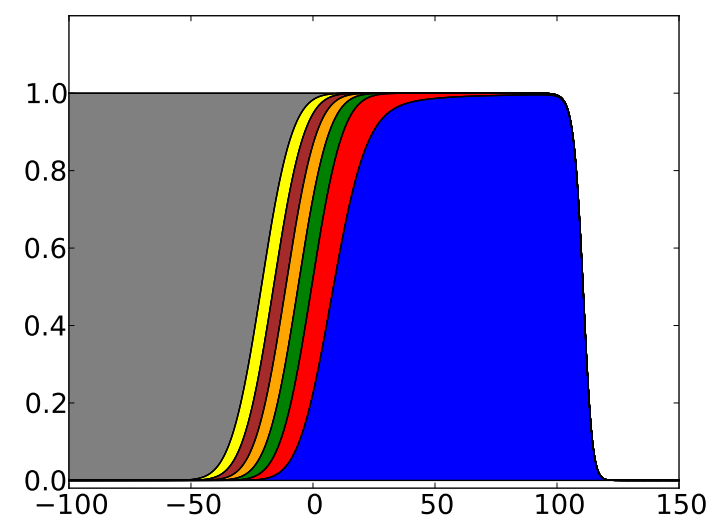

(e) Hutchinson $(\tau=0.3), t=50$

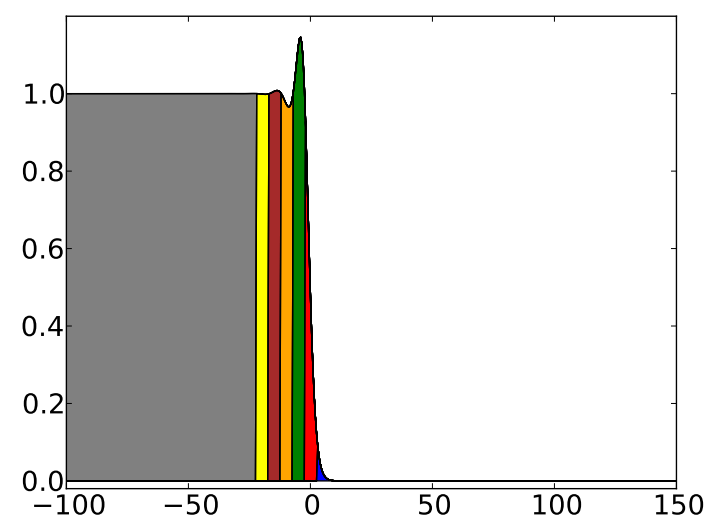

(b) Hutchinson $(\tau=1), t=0$

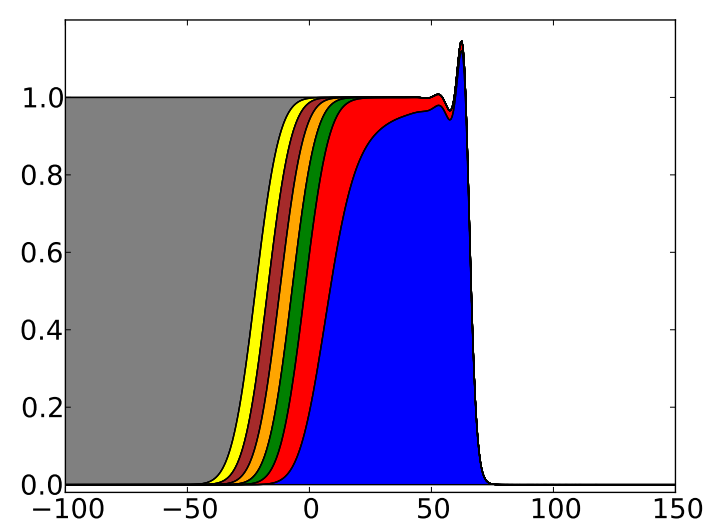

(d) Hutchinson $(\tau=1), t=30$

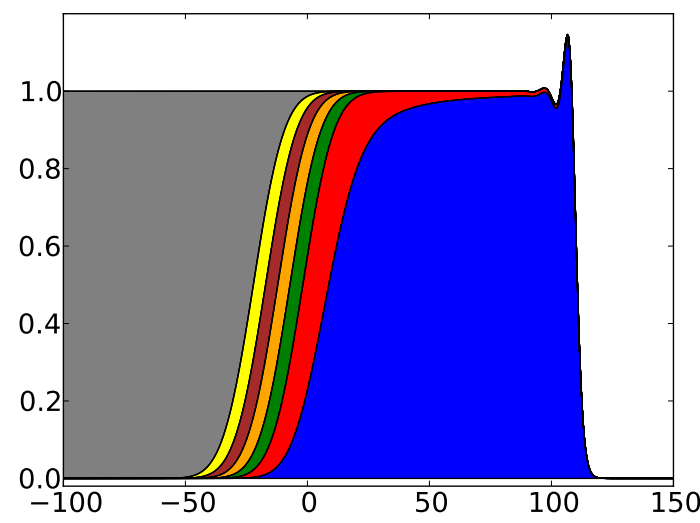

(f) Hutchinson $(\tau=1), t=100$

Figure 3: Inside dynamics of monotone and nonmonotone Hutchinson waves. Left: $c=c_{H}^{*}=2, \tau=0.3$; right: $c=c_{H}^{*}=2, \tau=1$. 
In the KPP case $F=F_{1}$, we considered the wave $\left(c^{*}, U_{c^{*}}\right)$ with minimal speed $c^{*}=2$ (Fig. 1 , left column). In the Kobayashi case $F=F_{2}$, we assumed that $\tau=10$, and we considered the case of the wave with minimal speed $c=c^{*}(10) \simeq 0.60$ (Fig. 2, left column) and the case of a wave with speed $c \simeq 1.11>\bar{c}(10) \simeq 0.66$, (Fig. 2, right column) where $\bar{c}(\tau)$ is defined in Proposition 1.4 (see formulas (3.1) and (3.2) below for the computation of $c^{*}(10)$ and formula (3.7) for the computation of $\left.\bar{c}(10)\right)$. We observe that, in all cases, the waves have a pulled structure: at large times, it tends to be made only of the rightmost initial component $v^{7}$. This indicates in particular that the wave with minimal speed is a pulled wave in the Kobayashi case.

However, it seems important to note that the red component $v^{6}$ stays at a non-negligible proportion during a large period of time in the Kobayashi case with $c=c^{*}(10)$. This shows that the transient transport phenomenon described by Proposition 1.4 leads to inside dynamics which are different from the dynamics of the KPP wave, even if the wave is not a pushed wave. From the point of view of populations genetics, this shows that the existence of a non-reproductive and motionless juvenile stage can slightly enhance genetic diversity in a colonization front (see Section 1.2), at least at the beginning of the colonization. Interestingly, when $c \simeq 1.11>\bar{c}(10) \simeq 0.66$, this phenomenon does not occur any more, as predicted by Proposition 1.4.

In the Hutchinson case $F=F_{3}$, we analyzed the waves with minimal speed $c=c_{H}^{*}=2$ with two different delays. Firstly, when $\tau=0.3<e^{-1}$, the results of [16] presented in Section 1.3 show that the wave is monotone (Fig. 3, left column). Second, when $\tau=1$, the results of [20] presented in Section 1.3 show that the wave is nonmonotone (Fig. 3, right column). In the case of the monotone wave $\tau=0.3$, the inside structure is very close to the KPP case, and in both cases, as predicted by Theorem 1.3, the waves have a pulled structure.

Lastly, in the Huxley case $F=F_{4}$, we analyzed the structure of the unique wave $\left(c_{4}, U_{4}\right)$ when $\tau=1$. Fig. 1 (right column) indicates that this is a pushed wave. We can observe that, at large times, all the initial components remain at constant positive proportions in the wave. Note that the proportion of the rightmost initial component $v^{7}$ is very small at large times, compared with the other cases. This last example emphasizes the pulled nature of the other waves (when $F=F_{k}, k=1,2,3$ ).

\subsection{Method for the numerical computation of the inside dynamics of the waves}

The method which is used here is comparable to that of [34]. However, since it was not described in [34], we give here all the technical details.

\subsubsection{Profile of the global wave}

In all cases $F=F_{k}, k=1, \ldots, 4$, we begin with the computation of the profile $U_{c}$ of the wave. To do so, we solved the Cauchy problem:

$$
\left\{\begin{array}{l}
\partial_{t} u(t, x)=\partial_{x x} u(t, x)+F(u(t-\tau, x), u(t, x)), \quad t>0, x \in \mathbb{R}, \\
u(t, x)=u_{0}(x), t \in[-\tau, 0], x \in \mathbb{R}
\end{array}\right.
$$

where $u_{0}$ is either a step function:

$$
u_{0}(x)=1 \text { for } x \leq 0 \text { and } u_{0}(x)=0 \text { for } x>0,
$$

or an exponentially decaying function:

$$
u_{0}(x)=1 \text { for } x \leq 0 \text { and } u_{0}(x)=e^{-\lambda_{c}^{-} x} \text { for } x>0,
$$

where $\lambda_{c}^{-}$is the smallest root of the equation $\lambda^{2}-c \lambda+\beta e^{-\lambda c \tau}=0$ and corresponds to the exponent in the asymptotic behavior of the wave with speed $c>c^{*}(\tau)$, in the Kobayasi case (see Section 3.2).

In the KPP case without delay $\left(\tau=0, F=F_{1}\right)$, it is known that the solution of the equation (2.1) with the initial condition $(2.2)$ converges to the traveling wave with speed $c^{*}(0)=2$, in the following sense:

$$
u(t, x) \rightarrow U_{c^{*}(0)}\left(x-c^{*}(0) t+m(t)\right), \text { uniformly in } x \text { as } t \rightarrow+\infty,
$$


where $m(t)=(3 / 2) \ln (t)+O(1)$ is sublinear $[8,19]$.

In the Kobayashi case $\left(\tau>0, F=F_{2}\right)$, when $u_{0}$ satisfies $(2.2), c^{*}(\tau)$ is the asymptotic speed of propagation of the solution $u(t, x)$ of $(2.1)$ (theorem 2.12 in [38]). The convergence of the solution of (2.1) towards the traveling wave $\left(c^{*}(\tau), U_{c^{*}(\tau)}\right)$ with speed $c^{*}(\tau)$ is not proved but can be observed numerically. Similarly, when $u_{0}$ satisfies $(2.3)$, we observed numerically that $u(t, x)$ converges to a traveling wave $\left(c, U_{c}\right)$ with the speed $c>c^{*}(\tau)$ associated with $\lambda_{c}^{-}$. In our computation, we took $\lambda_{c}^{-}=0.17$, which leads to $c \simeq 1.11>\bar{c}(10) \simeq 0.66$.

In the Hutchinson and Huxley cases $\left(\tau>0, F=F_{3}\right.$ and $\left.F=F_{4}\right)$, the profiles of the waves $\left(c_{H}^{*}=\right.$ $\left.2, U_{c_{H}^{*}}\right)$ and $\left(c_{4}, U_{4}\right)$ respectively, have been computed by solving $(2.1)$ with the initial condition $(2.2)$. In both cases, we observed the convergence of the solution towards the sought traveling wave.

\subsubsection{Dynamics of the inside components}

Once the profile $U_{c}$ of the wave has been computed, we solve, for each component $v^{i}(1 \leq i \leq N=7)$ the following system:

$$
\left\{\begin{array}{rlrl}
\partial_{t} u(t, x) & =\partial_{x x} u(t, x)+F_{k}(u(t-\tau, x), u(t, x)), & & t>0, x \in \mathbb{R}, \\
\partial_{t} v^{i}(t, x) & =\partial_{x x} v^{i}(t, x)+h\left[v^{i}, u, t, x\right] F_{k}(u(t-\tau, x), u(t, x)), & t>0, x \in \mathbb{R}, \\
u(t, x) & =U_{c}(x-c t), & t \in[-\tau, 0], x \in \mathbb{R}, \\
v^{i}(t, x) & =v_{0}^{i}(x-c t), & & t \in[-\tau, 0], x \in \mathbb{R} .
\end{array}\right.
$$

where $h\left[v^{i}, u, t, x\right]=v^{i}(t, x) / u(t, x)$ in the cases $F=F_{1}, F_{3}, F_{4}$ and $h\left[v^{i}, u, t, x\right]=v^{i}(t-\tau, x) / u(t-\tau, x)$ in the Kobayashi case $F=F_{2}$. In all cases, we observed that the condition

$$
u(t, x)=\sum_{i \in I} v^{i}(t, x) \text { for all } t>0 \text { and } x \in \mathbb{R}
$$

was perfectly fulfilled.

\subsubsection{Technical aspects}

The system (2.1) was solved on a bounded interval $[a, b]$ (see Table 1 ) with the boundary conditions $u(t, a)=1$ and $u(t, b)=0$. The profile $U_{c}$ was computed from the solution $u(t, x)$ at a sufficiently large time $T_{U}$ (see Table 1 ).

The system (2.4) was also solved on $[a, b]$ with the boundary conditions $u(t, a)=1, u(t, b)=0$, $v^{1}(t, a)=1, v^{i}(t, a)=0$ for $i=2, \ldots, 7$ and $v^{i}(t, b)=0$ for $i=1, \ldots, 7$. For the computation of the initial conditions, we used the above-computed profile $U_{c}$, which was shifted to the left so that $U_{c}(0)=1 / 2$.

The simulations are based on the Finite Element Method with piecewise linear and continuous basis functions. The discretization space is uniform with a step $\delta x=0.1$. We used a backward Euler method with a time step $\delta t=0.1$. The nonlinearity has been treated with a Newton-Raphson algorithm applied to the variational formulation of the problems $(2.1)$ and $(2.4)$ on the bounded interval $[a, b]$. The criterion of convergence was equal to $10^{-5}$. We used the software Freefem ++ to perform these simulations.

The numerical error can be assessed as follows. Let $u$ be the solution of the system (2.4), and let $u_{h}$ be the piecewise linear and continuous function computed by the Finite Element Method. It is classical that the $H^{1}$-error $\left\|u-u_{h}\right\|_{H^{1}([a, b])}$ is controlled by $\delta x\left\|u^{\prime \prime}\right\|_{L^{2}([a, b])}$. Thanks to Freefem++, we are able to compute an approximation of this error by using $\delta x\left\|u_{h}^{\prime \prime}\right\|_{L^{2}([a, b])}$, see Table 1 . 


\begin{tabular}{|l|c|c|c|c|}
\hline Simulation & $\delta x\left\|u_{h}^{\prime \prime}\right\|_{L^{2}([a, b])}$ & $\mathrm{a}$ & $\mathrm{b}$ & $T_{U}$ \\
\hline$F=F_{1}$ & $2.710^{-3}$ & -500 & 1000 & 100 \\
\hline$F=F_{2}, u_{0}$ satisfies $(2.2)$ & $9.410^{-4}$ & -500 & 1000 & 500 \\
\hline$F=F_{2}, u_{0}$ satisfies $(2.3)$ & $2.210^{-3}$ & -500 & 1000 & 500 \\
\hline$F=F_{3}, \tau=0.3$ & $1.910^{-2}$ & -500 & 2500 & 500 \\
\hline$F=F_{3}, \tau=1$ & $1.910^{-2}$ & -500 & 2500 & 500 \\
\hline$F=F_{4}$ & $5.510^{-3}$ & -500 & 1000 & 1000 \\
\hline
\end{tabular}

Table 1

\section{Proofs}

\subsection{Proof of Theorem 1.3}

\subsubsection{Kobayashi case}

Assume that $F$ satisfies (1.4)-(1.5) and let $\tau>0$. Let $\left(c, U_{c}\right)$ be a traveling wave solving (1.7), with speed $c>c^{*}(\tau)$. From Lemma 2.5 in [38], the minimal speed $c^{*}(\tau)$ is characterized by

$$
c^{*}(\tau)=\inf \left\{c>0, \exists \lambda>0 \text { with } \Delta_{c}(\lambda)=0\right\},
$$

where

$$
\Delta_{c}(\lambda):=\lambda^{2}-c \lambda+\beta e^{-\lambda c \tau}
$$

with $\beta>0$ defined by (1.5) (iv).

Assume that $v_{0}$ is supported in $(-\infty, M]$ for some $M \in \mathbb{R}$, and consider the solution $v$ of (1.10). Let us set:

$$
w(t, x)=e^{-\lambda^{*}(\tau)\left(x-c^{*}(\tau)(t+\tau)-M\right)}, \text { for } t \geq-\tau, x \in \mathbb{R},
$$

where $\lambda^{*}(\tau)$ is the unique (double) positive root of the equation $\Delta_{c^{*}(\tau)}(\lambda)=0$. In order to prove part 1 of Theorem 1.3, we are going to show that $w$ is a super-solution of the equation (1.10) satisfied by $v$. A straightforward computation shows that, for all $t \geq 0$ and $x \in \mathbb{R}$,

$$
\partial_{t} w(t, x)-w_{x x}(t, x)-\beta w(t-\tau, x)=-w(t, x)\left(\left(\lambda^{*}(\tau)\right)^{2}-c^{*}(\tau) \lambda^{*}(\tau)+\beta e^{-\lambda^{*}(\tau) c^{*}(\tau) \tau}\right) .
$$

From the definition of $\lambda^{*}(\tau)$, we get that

$$
\partial_{t} w(t, x)-w_{x x}(t, x)-\beta w(t-\tau, x)=0 \text { for all } t \geq 0 \text { and } x \in \mathbb{R} .
$$

From the assumptions (ii) and (iv) in (1.5), we have $f_{1}(r) \leq \beta r$ for all $r \in[0,1]$, and, from the assumptions (ii) and (v) in (1.5), $f_{2}(s) \leq 1$ for all $s \in[0,1]$. Thus, $F(r, s) \leq \beta r$ for all $(r, s) \in[0,1]^{2}$. In particular, since $u(t, x)=U_{c}(x-c t) \in[0,1]$ and $v(t, x) \geq 0$ for all $t \in[-\tau, \infty)$ and $x \in \mathbb{R}$, we get:

$$
\partial_{t} v(t, x)-\partial_{x x} v(t, x)=\frac{v(t-\tau, x)}{u(t-\tau, x)} F(u(t-\tau, x), u(t, x)) \leq \beta v(t-\tau, x), t>0, x \in \mathbb{R} .
$$

Thus, (3.3) implies that $w$ is a super-solution of the equation satisfied by $v$. On the other hand, $v(t, x)=$ $v_{0}(x-c t) \leq 1 \leq w(t, x)$ for $t \in[-\tau, 0]$ and $x \leq M$, while $v(t, x)=v_{0}(x-c t)=0 \leq w(t, x)$ for $t \in[-\tau, 0]$ and $x \geq M$. Therefore, the maximum principle (Proposition 2.2 in [38]) implies that

$$
v(t, x) \leq w(t, x) \text { for all } t \geq 0 \text { and } x \in \mathbb{R} .
$$

Since $c>c^{*}(\tau)$, this last inequality readily implies the result of part 1 of Theorem 1.3. 


\subsubsection{Hutchinson case}

Assume that $F$ satisfies (1.6). Let $\tau>0$, and let $\left(c, U_{c}\right)$ be a (monotone or nonmonotone) traveling wave solving (1.7), with $c \geq c_{H}^{*}=2$. Assume that $v_{0}$ is supported in $(-\infty, M]$ for some $M \in \mathbb{R}$, and consider the solution $v$ of (1.11). Since the wave $u(t, x)=U_{c}(x-c t)$ and the component $v$ are positive for all $t>0$ and $x \in \mathbb{R}$, we have:

$$
\partial_{t} v(t, x)-\partial_{x x} v(t, x)-v(t, x) \leq 0, t>0, x \in \mathbb{R} .
$$

The classical parabolic maximum principle then implies that

$$
v(t, x) \leq\left(\sup _{\mathbb{R}} v_{0}\right) \frac{e^{t}}{\sqrt{4 \pi t}} \int_{-\infty}^{M} e^{\frac{-(x-y)^{2}}{4 t}} d y
$$

for all $t>0$ and $x \in \mathbb{R}$. Let $A \in \mathbb{R}$, we have:

$$
v(t, x+A+2 t) \leq \frac{\sup _{\mathbb{R}} v_{0}}{\sqrt{4 \pi t}} e^{-(x+A)} \int_{-\infty}^{M} e^{y} e^{\frac{-(x+A-y)^{2}}{4 t}} d y,
$$

which in turns implies that:

$$
v(t, x+A+2 t) \leq \frac{\sup _{\mathbb{R}} v_{0}}{\sqrt{4 \pi t}} e^{-(x+A-M)} .
$$

Finally, for all $x \geq 0$, we have:

$$
v(t, x+A+2 t) \leq \frac{\sup _{\mathbb{R}} v_{0}}{\sqrt{4 \pi t}} e^{M-A},
$$

which implies the result of part 2 of Theorem 1.3.

\subsection{Proof of Proposition 1.4 and Lemma 1.5}

Assume that $F$ satisfies (1.4)-(1.5) and let $\tau>0$. Let $\left(c, U_{c}\right)$ be a traveling wave solving (1.7), with speed $c>c^{*}(\tau)$. The results in [47] (Theorem 2.5 in Chapter 11) show that the wave has the following asymptotic behavior:

$$
\left\{\begin{array}{l}
U_{c}(x)=A e^{-\lambda_{c}^{-} x}+O\left(e^{-\left(\lambda_{c}^{-}+\delta\right) x}\right) \\
U_{c}^{\prime}(x)=-\lambda_{c}^{-} A e^{-\lambda_{c}^{-} x}+O\left(e^{-\left(\lambda_{c}^{-}+\delta\right) x}\right)
\end{array} \text { as } x \rightarrow+\infty,\right.
$$

where $\delta>0, A>0$ and $\lambda_{c}^{-}$is the smallest root of the equation $\Delta_{c}(\lambda)=0$. We deduce from (1.14) and (3.4) that

$$
\lim _{x \rightarrow+\infty} V(x):=c+2 \lim _{x \rightarrow+\infty} \frac{U_{c}^{\prime}(x)}{U_{c}(x)}=c-2 \lambda_{c}^{-} .
$$

The next lemmas give precise estimates of the exponents $\lambda^{*}(\tau)$ and $\lambda_{c}^{-}$.

Lemma 3.1. There holds

$$
\lambda^{*}(\tau)>\frac{c^{*}(\tau)}{2} .
$$

Proof. Since $\lambda^{*}(\tau)$ is the unique root of $\Delta_{c^{*}(\tau)}(\lambda)=0$, we have $\Delta_{c^{*}(\tau)}^{\prime}\left(\lambda^{*}(\tau)\right)=0$, that is

$$
2 \lambda^{*}(\tau)-c^{*}(\tau)-\beta c^{*}(\tau) \tau e^{-\lambda^{*}(\tau) c^{*}(\tau) \tau}=0 .
$$

Finally,

$$
\lambda^{*}(\tau)=\frac{c^{*}(\tau)}{2}\left(1+\beta \tau e^{-\lambda^{*}(\tau) c \tau}\right)>\frac{c^{*}(\tau)}{2},
$$

from the assumption (1.5)-(iv). 
Lemma 3.2. There exists a real number $\bar{c}(\tau) \in\left(c^{*}(\tau),+\infty\right)$ such that

$$
\lambda_{c}^{-}>\frac{c}{2} \text { for all } c \in\left(c^{*}(\tau), \bar{c}(\tau)\right) \text { and } \lambda_{c}^{-} \leq \frac{c}{2} \text { for all } c \geq \bar{c}(\tau) .
$$

Proof. For any $c>c^{*}(\tau)$, the equation $\Delta_{c}(\lambda)=0$ admits two distinct roots, $\lambda_{c}^{-}$and $\lambda_{c}^{+}$, with $0<\lambda_{c}^{-}<$ $\lambda_{c}^{+}$, and

$$
\left\{\begin{array}{l}
\Delta_{c}(\lambda)>0 \text { if } \lambda \in\left[0, \lambda_{c}^{-}\right), \\
\Delta_{c}(\lambda) \leq 0 \text { if } \lambda \in\left[\lambda_{c}^{-}, \lambda_{c}^{+}\right], \\
\Delta_{c}(\lambda)>0 \text { if } \lambda \in\left(\lambda_{c}^{+},+\infty\right) .
\end{array}\right.
$$

If $\Delta_{c}(c / 2) \leq 0$ (this is the case for instance if $c$ is large enough), then we necessarily have $\lambda_{c}^{-} \leq c / 2$. Conversely, if $\Delta_{c}(c / 2)>0$, then either $c / 2<\lambda_{c}^{-}$or $c / 2>\lambda_{c}^{+}$. On the other hand, it follows from the convexity of the function $\lambda \mapsto \Delta_{c}(\lambda)$ that

$$
\Delta_{c}^{\prime}\left(\lambda_{c}^{+}\right)=2 \lambda_{c}^{+}-c-\beta c \tau e^{-\lambda_{c}^{+} c \tau}>0
$$

thus $\lambda_{c}^{+}>c / 2$. Thus $\Delta_{c}(c / 2)>0$ implies $c / 2<\lambda_{c}^{-}$. Hence,

$$
\lambda_{c}^{-}>\frac{c}{2} \text { if and only if } \Delta_{c}\left(\frac{c}{2}\right)=\beta e^{-\frac{c^{2} \tau}{2}}-\frac{c^{2}}{4}>0 .
$$

Furthermore, it is obvious to see that there exists a unique $\bar{c}(\tau)>0$ such that

$$
\Delta_{\bar{c}(\tau)}\left(\frac{\bar{c}(\tau)}{2}\right)=\beta e^{-\frac{\bar{c}(\tau)^{2} \tau}{2}}-\frac{\bar{c}(\tau)^{2}}{4}=0 .
$$

Additionally, we have $\Delta_{c}(c / 2)>0$ for $c \in[0, \bar{c}(\tau))$ and $\Delta_{c}(c / 2) \leq 0$ for $c \geq \bar{c}(\tau)$. From (3.6), we obtain that

$$
\lambda_{c}^{-}>\frac{c}{2} \text { if and only if } c<\bar{c}(\tau)
$$

Lastly, since $\lambda^{*}(\tau)$ is the unique root of $\Delta_{c^{*}(\tau)}(\lambda)=0$, Lemma 3.1 implies that

$$
\Delta_{c^{*}(\tau)}\left(\frac{c^{*}(\tau)}{2}\right)>0 .
$$

This in turn yields $\bar{c}(\tau)>c^{*}(\tau)$ and it guarantees the existence of a continuum of traveling wave solutions of (1.7) with the asymptotic behavior (3.4) and $\lambda_{c}^{-}>c / 2$, i.e., for all $c \in\left(c^{*}(\tau), \bar{c}(\tau)\right)$. The proof of Lemma 3.2 is thereby complete.

The conclusion of Proposition 1.4 follows from (3.5) and Lemma 3.2.

Proof of Lemma 1.5. From the characterization of $c^{*}(\tau)$ and $\bar{c}(\tau)$ given in (3.1) and in the previous lemma, it is straightforward to observe that $\lim _{\tau \rightarrow 0^{+}} c^{*}(\tau)=\lim _{\tau \rightarrow 0^{+}} \bar{c}(\tau)=2 \sqrt{\beta}$ and $\lim _{\tau \rightarrow+\infty} c^{*}(\tau)=$ $\lim _{\tau \rightarrow+\infty} \bar{c}(\tau)=0$.

Acknowledgements. The research leading to these results has received funding from the French ANR within the projects MACBI and PREFERED. A part of this work was done while J. Garnier was visiting the Lewis Research Group at the University of Alberta.

\section{References}

[1] M. Aguerrea, S. Trofimchuk, G. Valenzuela. Uniqueness of fast travelling fronts in reaction-diffusion equations with delay. Proc. Royal Soc. A, 464 (2008), 2591-2608. 
[2] S. Ai. Traveling wave fronts for generalized Fisher equations with spatio-temporal delays. J. Diff. Equations, 232 (2007), 104-133.

[3] D. G. Aronson, H. G. Weinberger. Nonlinear diffusion in population genetics, combustion and nerve propagation. In Partial Differential Equations and Related Topics, volume 446 of Lectures Notes Math, 5-49. Springer, New York, 1975.

[4] D. G. Aronson, H. G. Weinberger. Multidimensional nonlinear diffusion arising in population genetics. Adv. Math., 30 (1978), 33-76.

[5] F. Austerlitz, S. Mariette, N. Machon, P. H. Gouyon, B. Godelle. Effects of colonization processes on genetic diversity: differences between annual plants and tree species. Genetics, 154 (2000), 1309-1321.

[6] L. Berec, E. Angulo, F. Courchamp. Multiple Allee effects and population management. Trends Ecol. Evol., 22 (2007), $185-191$.

[7] J. Billingham, D. J. Needham. The development of traveling waves in quadratic and cubic autocatalysis with unequal diffusion rates. I. Permanent form of traveling waves. Phil. Trans. Royal Soc. A, 334 (1991), 1-24.

[8] M. Bramson. Convergence of solutions of the Kolmogorov equation to travelling waves. Mem. Amer. Math. Soc., 44 (1983).

[9] N. F. Britton. Reaction-Diffusion Equations and their Applications to Biology. Academic Press, London, 1986.

[10] J. P. Eckmann, C. E. Wayne. The nonlinear stability of front solutions for parabolic partial differential equations. Comm. Math. Phys., 161 (1994), 323-334.

[11] T. Faria, S. Trofimchuk. Nonmonotone travelling waves in a single species reaction-diffusion equation with delay. J. Diff. Equations, 228 (2006), 357-376.

[12] P. C. Fife. Mathematical Aspects of Reacting and Diffusing Systems, volume 28 of Lecture Notes in Biomathematics. Springer-Verlag, 1979.

[13] P. C. Fife, J. McLeod. The approach of solutions of nonlinear diffusion equations to traveling front solutions. Arch. Ration. Mech. Anal., 65 (1977), 335-361.

[14] J. Garnier, T. Giletti, F. Hamel, L. Roques. Inside dynamics of pulled and pushed fronts. J. Math. Pures Appl., 98 (2012), 428-449.

[15] J. Garnier, L. Roques, F. Hamel. Success rate of a biological invasion in terms of the spatial distribution of the founding population. B. Math. Biol., 74 (2012), 453-473.

[16] A. Gomez, S. Trofimchuk. Monotone traveling wavefronts of the KPP-Fisher delayed equation. J. Diff. Equations, 250 (2011), 1767-1787.

[17] P. Grindrod. Theory and Applications of Reaction-Diffusion Equations. Clarendon Press, 1996.

[18] O. Hallatschek, D. R. Nelson. Gene surfing in expanding populations. Theor. Popul. Biol., 73 (2008), 158-170.

[19] F. Hamel, J. Nolen, J.-M. Roquejoffre, L. Ryzhik. A short proof of the logarithmic Bramson correction in Fisher-KPP equations. Netw. Heterog. Media, In press (2013).

[20] K. Hasik, S. Trofimchuk. Slowly oscillating wavefronts of the KPP-Fisher delayed equation. arXiv, arXiv:1206.0484 (2012).

[21] A. L. Hodgkin, A. F. Huxley. A quantitative description of membrane current and its application to conduction and excitation in nerve. J. Physiology, 117 (1952), 500-544.

[22] G. E. Hutchinson. Circular causal systems in ecology. Ann. New York Acad. Sci., 50 (1948), 221-246.

[23] J. I. Kanel. Certain problems of burning-theory equations. Sov. Math. Doklady, 2 (1961), 48-51.

[24] K. Kobayashi. On the semilinear heat equations with time-lag. Hiroshima Math. J., 7 (1977), 459-472.

[25] A. N. Kolmogorov, I. G. Petrovsky, N. S. Piskunov. Étude de l'équation de la diffusion avec croissance de la quantité de matière et son application à un problème biologique. Bull. Univ. État Moscou, Sér. Int. A, 1 (1937), 1-26.

[26] M. K. Kwong, J. Ou. Existence and nonexistence of monotone traveling waves for the delayed Fisher equation. J. Diff. Equations, 249 (2010), 728-745.

[27] K.-S. Lau. On the nonlinear diffusion equation of Kolmogorov, Petrovsky and Piscounov. J. Diff. Equations, 59 (1985), $44-70$.

[28] M. A. Lewis, P. Kareiva. Allee dynamics and the spread of invading organisms. Theor. Popul. Biol., 43 (1993), $141-158$.

[29] M. A. Lewis, P. Van Den Driessche. Waves of extinction from sterile insect release. Math. Biosci., 116 (1993), $221-247$.

[30] X. Liang, X.-Q. Zhao. Asymptotic speeds of spread and traveling waves for monotone semiflows with applications. Comm. Pure Appl. Math., 60 (2007), 1-40.

[31] S. Ma. Traveling waves for non-local delayed diffusion equations via auxiliary equations. J. Diff. Equations, 237 (2007), 259-277.

[32] J. D. Murray. Mathematical Biology. Third Edition. Interdisciplinary Applied Mathematics 17, Springer-Verlag, New York, 2002.

[33] S. Pan. Asymptotic behavior of travelling fronts of the delayed Fisher equation. Nonlinear Anal. Real World Appl., 10 (2009), 1173-1182.

[34] L. Roques, J. Garnier, F. Hamel, E K. Klein. Allee effect promotes diversity in traveling waves of colonization. Proc. Natl. Acad. Sci. USA, 109 (2012), 8828-8833.

[35] L. Roques, F. Hamel, J. Fayard, B. Fady, E K. Klein. Recolonisation by diffusion can generate increasing rates of spread. Theor. Popul. Biol., 77 (2010), 205-212.

[36] D. H. Sattinger. On the stability of waves of nonlinear parabolic systems. Adv. Math., 22 (1976), $312-355$.

[37] D. H. Sattinger. Weighted norms for the stability of traveling waves. J. Diff. Equations, 25 (1977), $130-144$. 
[38] K. W. Schaaf. Asymptotic behavior and traveling wave solutions for parabolic functional differential equations. Trans. Amer. Math. Soc., 302 (1987), 587-615.

[39] N. Shigesada, K. Kawasaki. Biological Invasions: Theory and Practice. Oxford Series in Ecology and Evolution, Oxford: Oxford University Press, 1997.

[40] A. N. Stokes. On two types of moving front in quasilinear diffusion. Math. Biosci., 31 (1976), 307-315.

[41] E. Trofimchuk, P. Alvarado, S. Trofimchuk. On the geometry of wave solutions of a delayed reaction-diffusion equation. J. Diff. Equations, 246 (2009), 1422-1444.

[42] E. Trofimchuk, M. Pinto, S. Trofimchuk. Pushed traveling fronts in monostable equations with monotone delayed reaction. arXiv:1111.5161v1.

[43] K. Uchiyama. The behaviour of solutions of some non-linear diffusion equations for large time. J. Math. Kyoto Univ., 18 (1978), 453-508.

[44] M. O. Vlad, L. L. Cavalli-Sforza, J. Ross. Enhanced (hydrodynamic) transport induced by population growth in reactiondiffusion systems with application to population genetics. Proc. Natl. Acad. Sci. USA, 101 (2004), 10249-10253.

[45] Z.-C. Wang, W.-T. Li, S. Ruan. Travelling wave fronts of reaction-diffusion systems with spatio-temporal delays. J. Diff. Equations, 222 (2006), 185-232.

[46] Z.-C. Wang, W.-T. Li, S. Ruan. Existence and stability of traveling wave fronts in reaction advection diffusion equations with nonlocal delay. J. Diff. Equations, 238 (2007), 153-200.

[47] J. Wu. Theory and Applications of Partial Functional Differential Equations, volume 119 of Applied Mathematical Sciences. Springer-Verlag, New York, 1996.

[48] J. Wu, X. Zou. Traveling wave fronts of reaction-diffusion systems with delay. J. Dyn. Diff. Equations, 13 (2001), 651-687.

[49] X. Zou. Delay induced traveling wave fronts in reaction diffusion equations of fisher-kpp type. J. Comput. Appl. Math., 146 (2002), 309-321. 